\title{
Direct Injection Tunnel Spectroscopy of a p-n Junction
}

\section{Citation}

Likovich, Edward M., Kasey J. Russell, Venkatesh Narayanamurti, Hong Lu, and Arthur C. Gossard. 2009. Direct injection tunnel spectroscopy of a p-n junction. Applied Physics Letters 95(2): 022106.

\section{Published Version}

doi:10.1063/1.3177191

\section{Permanent link}

http://nrs.harvard.edu/urn-3:HUL.InstRepos:3606234

\section{Terms of Use}

This article was downloaded from Harvard University's DASH repository, and is made available under the terms and conditions applicable to Open Access Policy Articles, as set forth at http:// nrs.harvard.edu/urn-3:HUL.InstRepos:dash.current.terms-of-use\#OAP

\section{Share Your Story}

The Harvard community has made this article openly available.

Please share how this access benefits you. Submit a story.

Accessibility 


\title{
Direct injection tunnel spectroscopy of a p-n junction
}

\author{
Edward M. Likovich, Kasey J. Russell, ${ }^{*}$ and Venkatesh Narayanamurti \\ School of Engineering and Applied Sciences, \\ Harvard University, Cambridge, Massachusetts 02138 \\ Hong $\mathrm{Lu}$ and Arthur C. Gossard \\ Materials Department, University of California, Santa Barbara, California 93106
}

(Dated: December 22, 2009)

\begin{abstract}
We demonstrate spectroscopic measurements on an InGaAs p-n junction using direct tunnel injection of electrons. In contrast to the metal-base transistor design of conventional Ballistic Electron Emission Spectroscopy (BEES), the base layer of our device is comprised of a thin, heavily doped p-type region. By eliminating the metal base layer and tunneling directly into the semiconductor, we observe a significant increase in collector current compared to conventional BEES measurements. Such an approach could enable the study of systems and processes that have thus far been difficult to probe with the low electron collection efficiency of conventional BEES, such as luminescence from single buried quantum dots.

PACS numbers:
\end{abstract}

*Electronic address: krussell@post.harvard.edu 
Ballistic Electron Emission Spectroscopy (BEES) has been shown to be a useful probe of electron transport through buried metal-semiconductor interfaces $[1,2]$, semiconductor heterojunctions [3], and quantum dot structures [4]. Its optical counterpart, Ballistic Electron Emission Luminescence (BEEL), has enabled the study of luminescence from buried structures $[5,6]$, but thus far the spectroscopic study of luminescence from a single buried structure such as a quantum dot has remained elusive. The primary obstacle to such an experiment is the low collector current attainable in conventional BEES/BEEL techniques, which typically utilize a thin metal layer for the base electrode [7, 8]. Electrons tunnel into the base metal, and, except in select systems such as epitaxial Bi on $\mathrm{Si}$ [9], the vast majority $(>99.9 \%)$ are unable to traverse it and enter the semiconductor before they scatter and thermalize to the chemical potential of the base layer. For systems with an optically-active collector made of a III-V material such as GaAs, even epitaxial base layers have shown significant scattering [10].

Here we report spectroscopic results from a structure, shown in Figure 1b, in which the base electrode is instead formed by a thin, heavily-doped surface region of the opposite polarity from the semiconductor collector [11]. By replacing the metal base layer with a thin layer of p-type semiconductor, several electron scattering processes are eliminated. The first is scattering while traversing the base layer, the probability of which is given by $P_{\lambda}=\exp [-d / \lambda]$, where $d$ is the thickness of the metal base region and $\lambda$ is the ballistic mean free path of an electron in the metal layer [12]. For a typical 10nm to 20nm-thick base layer of $\mathrm{Ag}, \mathrm{Au}$ [12], or $\operatorname{ErAs}[13], P_{\lambda} \sim 0.1$. In addition, in metal-base systems the large potential step at the metal-semicondutor interface leads to a low probability of transmission without reflection, $P_{Q M} \sim 0.25$ [14]. In our system, in contrast, the potential energy varies smoothly between the base and collector layers, resulting in a significantly lower probability of reflection. Finally, in metal-base systems such as Au/GaAs, the probability of traversing the metal/semiconductor interface without scattering, $P_{B C}$, has been experimentally shown to be $\sim 0.1[15]$. In our device, the base and collector are made of the same material, so this scattering mechanism is not present. Thus, we would expect that for the same emitter structure and tunnel barrier, the collector current of a direct-injection device could be larger than that of a metal-base device by roughly $\left(P_{\lambda} P_{B C} P_{Q M}\right)^{-1} \sim 400$.

Parasitic effects in the direct-injection device may slightly reduce this estimate, but such effects should be negligible in an optimized structure. Minority carrier recombina- 

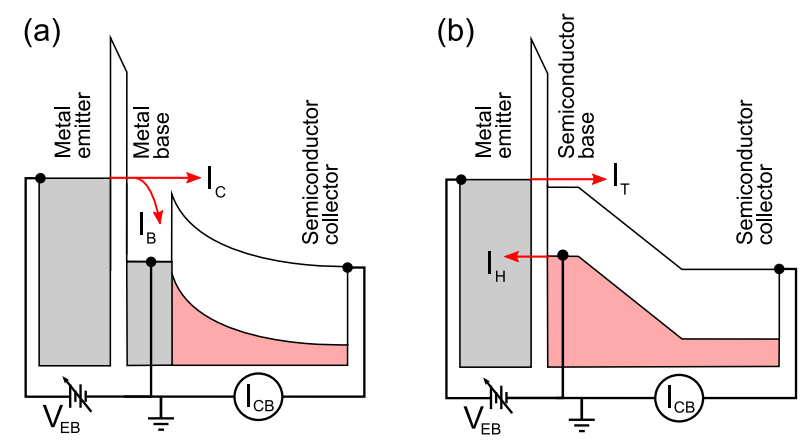

FIG. 1: (Color online) (a) Schematic energy band diagram of a conventional, metal-base device under conditions of a negative $V_{E B}$. Of the tunneling electrons, only a small fraction $I_{C}$ enter the collector; the vast majority scatter and lose energy within in the base metal, forming $I_{B}$. (b) Schematic energy band diagram of a direct tunnel injection device shown under similar bias conditions. Because the base electrode is formed by a doped semiconductor layer, there is less attenuation of the tunnel current, and $I_{C}$ can be orders of magnitude higher than in a metal-base device. The transfer ratio is ultimately limited by hole tunneling from the base into the emitter $\left(I_{H}\right)$.

tion within the p-type region is negligible for such a thin layer, as has been shown in bipolar transistors[16]. Additionally, surface recombination at the oxide-semiconductor interface should be insignificant because the emitter electric field will accelerate electrons away from the semiconductor surface.

The device used in this experiment was grown by molecular beam epitaxy, lattice-matched on a (100) n-type doped InP substrate. In order from surface to substrate (base to collector), the layers were: $50 \mathrm{~nm} \operatorname{In}_{0.53} \mathrm{Ga}_{0.47} \mathrm{As}$ (p-type doped $1 \times 10^{19} \mathrm{~cm}^{-3}$ with $\mathrm{Be}$ ); $300 \mathrm{~nm}$ $\mathrm{In}_{0.53} \mathrm{Ga}_{0.47} \mathrm{As}$; and $500 \mathrm{~nm} \operatorname{In}_{0.53} \mathrm{Ga}_{0.47} \mathrm{As}$ (n-type doped $3 \times 10^{18} \mathrm{~cm}^{-3}$ with $\mathrm{Si}$ ). Devices were fabricated by etching circular mesas of $150 \mu$ m diameter and $\sim 500 \mathrm{~nm}$ height. $\mathrm{Al}_{2} \mathrm{O}_{3}$ tunnel barriers were formed by fully oxidizing a thin $(\sim 1 \mathrm{~nm})$ sputtered Al film using a UV-O 3 treatment for $10 \mathrm{~min}$ at $100^{\circ} \mathrm{C}$. Emitter electrodes of $\sim 20 \mathrm{~nm} \mathrm{Al}$ or $\mathrm{Al} \backslash \mathrm{Ti} \backslash \mathrm{Au}(10 \mathrm{~nm} \backslash 10 \mathrm{~nm} \backslash 10 \mathrm{~nm})$ were sputtered on top of the oxide. Sputtered $\mathrm{Ti} \backslash \mathrm{Au}(10 \mathrm{~nm} \backslash 50 \mathrm{~nm})$ contacts were made to the emitter, base, and collector for electrical access to each of the layers. All measurements were made at $77 \mathrm{~K}$ in a common-base configuration.

Shown in Figure 2 are traces of collector current $I_{C}$ versus emitter-base bias $V_{E B}$ from 
two devices: one measured prior (U) and the other subsequent (A) to annealing for $1 \mathrm{~min}$ at $300^{\circ} \mathrm{C}$ in Ar. Similar annealing treatments have been shown to increase the resistivity,

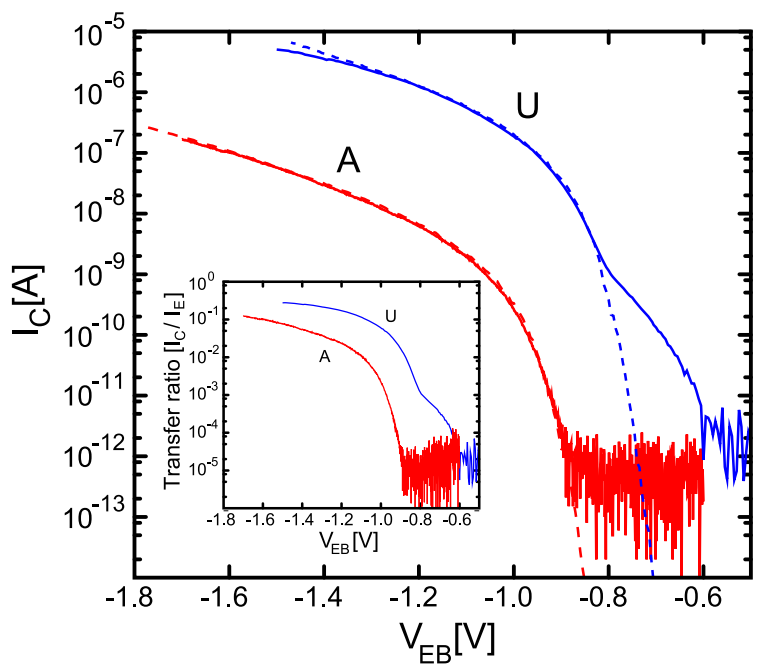

FIG. 2: (Color online) Collector current of an unannealed device (U) and a different, annealed device (A) as a function of emitter-base bias. Solid lines are measured data; dashed lines are simulation results for a $2.2 \mathrm{eV}$ barrier of thickness $1.76 \mathrm{~nm}(\mathrm{U})$ and $2.08 \mathrm{~nm}(\mathrm{~A})$. Simulations have been shifted in bias by less than $100 \mathrm{mV}$ for better agreement, as described in the text. Both samples show a sharp turn-on near $V_{E B}=-0.8 \mathrm{~V}$ (corresponding to the expected conduction band edge of InGaAs) and generally agree with the simulation. (Inset) Transfer ratios $\left(I_{C} / I_{E}\right)$ for the two devices. Both achieve values greater than $10 \%$.

stability, and break-down field of $\mathrm{Al}_{2} \mathrm{O}_{3}$ tunnel barriers [17]. As in a conventional BEES device, we expect the collector current $I_{C}$ to be negligible unless the tunneling electrons have sufficient energy to enter the collector. In our device, the chemical potential $\mu_{B}$ of the p-type base is approximately equal to the energy of the valence band $E_{V B}$, so the threshold in $I_{C}$ occurs when $q V_{E B}$ is approximately equal to the band gap of the semiconductor $(\sim 0.8 \mathrm{eV}$ at $77 \mathrm{~K}[18]$ ). In some devices, such as the unannealed device shown in Figure 2, we observed an additional threshold near $V_{E B}=-0.6 \mathrm{~V}$ whose origin is unknown but presumably related to defects or In non-uniformity within the base layer.

Also shown in Figure 2 are Monte Carlo simulations of $I_{C}$ calculated following the general 
format of Ref. [15]:

$$
\begin{aligned}
I_{C}= & e A \frac{\hbar}{m} \frac{n}{2 N} \sum k_{\perp} P_{o c c}(E) P_{v a c}(E) \\
& \times P_{\text {dos }}\left(k_{\|}, k_{\perp}\right) P_{\text {tunnel }}\left(k_{\perp}\right),
\end{aligned}
$$

where $e$ is the electron charge; $A$, the device area; $m$, the electron effective mass; $n$, the emitter electron density; $N$, the sampling number $\left(10^{8}\right) ; \hbar k_{\perp}$, the momentum in the direction of tunneling (perpendicular to the surface); $P_{\text {occ }}(E)$, the occupation probability of a state with energy $E$ in the emitter; $P_{v a c}(E)$, the probability of vacancy of a state of energy $E$ in the collector; $P_{d o s}\left(k_{\|}, k_{\perp}\right)$, the probability that a state with momentum components $\hbar k_{\|}$and $\hbar k_{\perp}$ parallel and perpendicular to the layers, respectively, exists in the semiconductor; and $P_{\text {tunnel }}\left(k_{\perp}\right)$ is the tunneling probability calculated using the scattering matrix formalism[19].

To achieve agreement with experimental data, we varied three simulation parameters: tunnel barrier height, tunnel barrier thickness, and $\mu_{B}$ (relative to the semiconductor valence band). For both the annealed and unannealed devices, we obtained best agreement with a $2.2 \mathrm{eV}$ barrier height. The resulting value for the thickness of the annealed barrier $(2.08 \mathrm{~nm})$ was slightly larger than that of the unannealed barrier $(1.76 \mathrm{~nm})$, consistent with the reported effects of annealing [17]. As expected, $\mu_{B}$ was found to be within $100 \mathrm{meV}$ of $E_{V B}: \mu_{B}=E_{V B}+25 \mathrm{meV}$ for the unannealed device and $\mu_{B}=E_{V B}-90 \mathrm{meV}$ for the annealed device. A drawback to the direct injection approach is that $\mu_{B}$ is a sensitive function of surface Fermi-level pinning and base doping and thus could be altered by annealing. However, we did not measure a sufficient number of devices to enable a systematic study of the effects of annealing on $\mu_{B}$.

As in conventional BEES, the collection probability in our structure can vary significantly depending on whether $k_{\|}$is conserved during transport. This becomes especially apparent for $q V_{E B}$ near the threshold of the conduction band minimum at $\mathrm{L}(\sim 550 \mathrm{meV}$ above the threshold of $\Gamma[18])$ in $\operatorname{In}_{0.53} \mathrm{Ga}_{0.47}$ As. The valley at $\mathrm{L}$ has a large density of states, but those states are located at $\{111\}$ and therefore have large projected $k_{\|}$in the (100) plane of the semiconductor surface. Because the tunneling probability depends exponentially on $k_{\perp}$, if $k_{\|}$is conserved, then $k_{\perp}$ must be reduced according to $k_{\perp}^{2}=2 m E / \hbar^{2}-k_{\|}^{2}$, and the current entering L will be exponentially supressed.

In conventional BEES, however, it is common for $k_{\|}$-conservation to be disrupted by scattering $[10,14]$. In such cases, the large density of states at L leads to an increase in $I_{C}$ at the threshold of $\mathrm{L}$ that is visible in derivative spectra. In Figure 3, we investigate this regime 
using the logarithmic derivative of $I_{C}$. In contrast to conventional BEES measurements, the

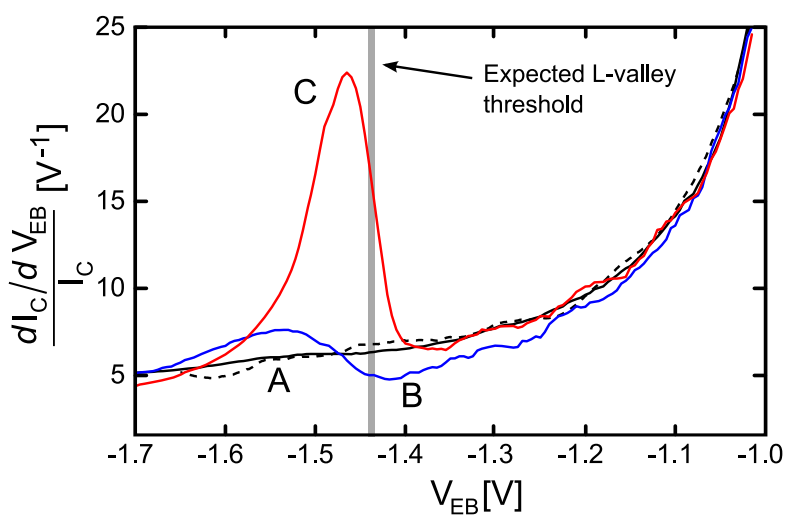

FIG. 3: (Color online) Logarithmic derivative of measured collector current (dashed line) from the annealed sample of Figure 2, as well as various simulations (solid lines, labeled). (A), Simulation with a $2.08 \mathrm{~nm}$-thick barrier (the same simulation as shown in Figure 2). No feature is visible at the L-valley threshold near -1.45V. (B), Simulation of a thinner tunnel barrier (0.80 nm); here the L-valley threshold is apparent. (C), Simulation with $2.08 \mathrm{~nm}$-thick barrier with no $k_{\|}$-conservation. The peak is from L-valley density of states.

measured $I_{C}$ in our device agrees with the simulation of $k_{\|}$-conserved transport (line A). The simulation with identical sample parameters but without $k_{\|}$-conservation (line $\mathrm{C}$ ) is clearly not a good description of transport in our device. Shown for comparison is a $k_{\|^{-}}$ conserved simulation with a thinner tunnel barrier (line B), in which the threshold at $\mathrm{L}$ is visible. Simulations of various barrier thicknesses (not shown) indicate that the threshold at $\mathrm{L}$ is visible in $k_{\|}$-conserved systems only for junctions thinner than $\sim 1.2 \mathrm{~nm}$. We cannot fabricate and measure a device with such a thin $\mathrm{Al}_{2} \mathrm{O}_{3}$ barrier because the voltage needed to reach the threshold at $\mathrm{L}$ would cause the emitter-base electric field to exceed the breakdown field of the barrier [17]. However, using the tip of a scanning tunneling microscope (STM) as an emitter electrode could allow probing at a variety of barrier thicknesses.

In summary, we have demonstrated spectroscopic measurements of direct tunnel injection into a p-n junction. The observed $I_{C}$ versus $V_{E B}$ agrees very well with simulations that include $k_{\|}$-conservation. Because this approach can provide higher $I_{C}$ than conventional BEES/BEEL, it represents a significant advance toward the spectroscopic study of luminescence from single buried quantum structures. 


\section{Acknowledgments}

This work was supported by NSF/NNIN through the use of their facilities at Harvard University's Center for Nanoscale Systems (CNS). Simulations were run on the Odyssey cluster supported by the Harvard University FAS Research Computing Group. E.M.L. acknowledges a U.S. Department of Homeland Security Graduate Research Fellowship.

[1] L. D. Bell and W. J. Kaiser, Phys. Rev. Lett. 61, 2368 (1988).

[2] W. J. Kaiser and L. D. Bell, Phys. Rev. Lett. 60, 1406 (1988).

[3] T. Sajoto, J. J. O’Shea, S. Bhargava, D. Leonard, M. A. Chin, and V. Narayanamurti, Phys. Rev. Lett. 74, 3427 (1995).

[4] C. V. Reddy, V. Narayanamurti, J. H. Ryou, and R. D. Dupuis, Appl. Phys. Lett. 80, 1770 (2002).

[5] I. Appelbaum, K. J. Russell, V. Narayanamurti, D. J. Monsma, C. M. Marcus, M. P. Hanson, A. C. Gossard, H. Temkin, and C. H. Perry, Appl. Phys. Lett. 82, 2960 (2003).

[6] I. Appelbaum, K. Russell, M. Kozhevnikov, V. Narayanamurti, M. Hanson, and A. Gossard, Appl. Phys. Lett. 84, 547 (2004).

[7] W. Yi, A. Stollenwerk, and V. Narayanamurti, Surf. Sci. Rep. 64, 169 (2009).

[8] J. Smoliner, D. Rakoczy, and M. Kast, Rep. Prog. Phys. 67, 1863 (2004).

[9] A. Bannani, C. Bobisch, and R. Moller, Science 315, 1824 (2007).

[10] K. Russell, I. Appelbaum, V. Narayanamurti, M. Hanson, and A. Gossard, PRB 71 (2005), ISSN 1098-0121.

[11] H. Kisaki, Proc. of the IEEE 61, 1053 (1973).

[12] S. M. Sze, C. R. Crowell, G. P. Carey, and E. E. LaBate, J. Appl. Phys. 37, 2690 (1966).

[13] K. J. Russell, V. Narayanamurti, I. Appelbaum, M. P. Hanson, and A. C. Gossard, PRB 74, $205330(2006)$.

[14] D. L. Smith and S. M. Kogan, Phys. Rev. B 54, 10354 (1996).

[15] I. Appelbaum and V. Narayanamurti, Phys. Rev. B 71, 045320 (2005).

[16] S. Sze and K. K. Ng, Physics of Semiconductor Devices (Wiley-Interscience, 2007), 3rd ed.

[17] E. Cimpoiasu, S. K. Tolpygo, X. Liu, N. Simonian, J. E. Lukens, K. K. Likharev, R. F. Kile, 
and Y. Zhu, J. Appl. Phys. 96, 1088 (2004).

[18] I. Vurgaftman, J. R. Meyer, and L. R. Ram-Mohan, J. Appl. Phys. 89, 5815 (2001).

[19] D. Y. K. Ko and J. C. Inkson, Phys. Rev. B 38, 9945 (1988). 